\title{
Metastatic Malignant Neoplasm in the Thyroid Gland
}

National Cancer Institute

\section{Source}

National Cancer Institute. Metastatic Malignant Neoplasm in the Thyroid Gland. NCI

Thesaurus. Code C7509.

A malignant neoplasm either directly extending to the thyroid gland from adjacent structures (e.g. pharynx, larynx, esophagus, cervical lymph nodes), or spreading to the thyroid gland from distant anatomic sites, most frequently kidney, lung, uterus, breast, and skin. The majority of cases are carcinomas, lymphomas, and melanomas. 